\title{
Author Correction: Lung regeneration by multipotent stem cells residing at the bronchioalveolar-duct junction
}

Qiaozhen Liu (D), Kuo Liu, Guizhong Cui, Xiuzhen Huang (D), Shun Yao, Wenke Guo, Zhen Qin, Yan Li®, Rui Yang, Wenjuan Pu (1), Libo Zhang (D), Lingjuan He (D), Huan Zhao, Wei Yu (D, Muxue Tang, Xueying Tian (1), Dongqing Cai, Yu Nie (1), Shengshou Hu, Tao Ren, Zengyong Qiao, Hefeng Huang, Yi Arial Zeng, Naihe Jing @i, Guangdun Peng (D),

Hongbin Ji(D) and Bin Zhou (D)

Correction to: Nature Genetics https://doi.org/10.1038/s41588-019-0346-6, published online 18 February 2019.

In the version of this article initially published, the following grant numbers and recipients were missing from the Acknowledgements: XDB19000000 to H.J. and B.Z.; 81430066 and 31621003 to H.J.; 2017 YFA0505500 to H.J.; and 15XD1504000 to H.J. The errors have been corrected in the HTML and PDF versions of the article.

Published online: 7 March 2019

https://doi.org/10.1038/s41588-019-0388-9

\section{Author Correction: Osteogenesis depends on commissioning of a network of stem cell transcription factors that act as repressors of adipogenesis}

Alexander Rauch (D), Anders K. Haakonsson (D), Jesper G. S. Madsen, Mette Larsen (D), Isabel Forss (D), Martin R. Madsen, Elvira L. Van Hauwaert, Christian Wiwie, Naja Z. Jespersen, Michaela Tencerova, Ronni Niels (D., Bjørk D. Larsen,

Richard Röttger, Jan Baumbach, Camilla Scheele, Moustapha Kassem and Susanne Mandrup

Correction to: Nature Genetics https://doi.org/10.1038/s41588-019-0359-1, published online 4 March 2019.

In the version of this article initially published, in the graph keys in Fig. 1i, the colors indicating 'Ob' and 'Ad' were red and blue, respectively, but should have been blue and red, respectively; the shapes indicating 'MUS' and 'BM' were a triangle and a square, respectively, but should have been a square and a triangle, respectively. The errors have been corrected in the HTML and PDF versions of the article. 